\title{
Adsorption of Ultra-High Crosslinked Resin on Waste Water from Benzoic Acid and Sorbic Acid
}

\author{
Bin Liu, Cheng-yuan $\mathrm{Hu}$ \\ Yancheng Environmental Monitoring Center of Jiangsu province, Jiangsu, 224000, China
}

\begin{abstract}
Aiming at the simulated production wastewater of benzoic acid and sorbic acid, the adsorption and desorption resource recovery system of ultra-high crosslinked resin ZH-01 was designed and developed, and the influence of $\mathrm{ZH}-01$ on the removal of benzoic acid and sorbic acid production wastewater was studied. The results showed that the removal rates of benzoic acid and sorbic acid were $94.74 \%$ and $94.9 \%$, respectively, and the removal rate of CODcr was as high as $91.2 \%$. In addition, the adsorption properties of ZH-01 on benzoic acid and sorbic acid were investigated. When sodium hydroxide was used for resin desorption, the effects of temperature, desorption agent ratio and desorption velocity on resin desorption were investigated. Benzoic acid and sorbic acid were recovered to the maximum extent, and the recycling of benzoic acid and sorbic acid in waste water was realized.

Keywords Ultra high crosslinked resin ZH-01; Simulated production wastewater with benzoic acid and sorbic acid; Resource recovery.

DOI: $10.7176 / \mathrm{JRDM} / 57-05$

Publication date:July $31^{\text {st }} 2019$

\section{Introduction}

Benzoic acid and sorbic acid, is acknowledged as the toxicity and the lowest in the world's most widely used food preservatives ${ }^{[1-3]}$, which primarily by inhibiting the growth of microorganisms which have the effect of antiseptic, also is widely used in the pharmaceutical and dye. Emission levels of benzoic acid and sorbic acid wastewater will produce serious pollution to the environment, at the same time produce certain harm to the health of human beings, not only more biological produce certain effect of environments ${ }^{[4-6]}$. Therefore, reasonable and efficient with benzoic acid and sorbic acid wastewater treatment in the wastewater treatment is particularly important and urgent. In this study, the optimal resin adsorption conditions of benzoic acid and sorbic acid wastewater were optimized [7-9] to systematically study the adsorption conditions and influencing factors of ultra-high crosslinked resin on benzoic acid and sorbic acid. According to the optimal adsorption conditions, the adsorption effects of ultra-high crosslinked resin on benzoic acid and sorbic acid were determined. At the same time, the optimal resin desorption conditions were studied.
\end{abstract}

\section{Experiment}

\subsection{Pretreatment of resin}

It was placed in a beaker for ultra-high crosslinked resin of ZH-01, and it was soaked with anhydrous ethanol, and was extracted with soxhlet extractor for $7 \sim 8 \mathrm{~h}$. Then it was vacuum dried at $333 \mathrm{~K}$ for $4 \mathrm{~h}$, and was followed by leaching resin with $4 \% \mathrm{HCl}$ and $4 \% \mathrm{NaOH}$ for $3 \mathrm{~h}$, respectively.

\subsection{Selection of measurement wavelength}

Accurately according to benzoic acid and sorbic acid $0.2000 \mathrm{~g}$ each , it was prepared for $200.0 \mathrm{mg} \cdot \mathrm{L}^{-1}$ solution in a volumetric flask with a constant volume of $1000 \mathrm{~mL}$, and the maximum absorption wavelength was determined.

\subsection{Standard curve drawing}

Benzoic acid and sorbic acid solutions with concentrations of $200.0 \mathrm{mg} \bullet \mathrm{L}^{-1}, 400.0 \mathrm{mg} \bullet \mathrm{L}^{-1}, 600.0 \mathrm{mg} \bullet \mathrm{L}^{-1}, 800.0$ $\mathrm{mg} \cdot \mathrm{L}^{-1}$ and $1000.0 \mathrm{mg} \cdot \mathrm{L}^{-1}$ were prepared, respectively, for $100.0 \mathrm{~mL}$. The absorbance is then measured at wavelength lambda $225 \mathrm{~nm}$ and $255 \mathrm{~nm}$ to produce the standard curve for benzoic acid and sorbic acid solutions $[14]$.

\subsection{CODcr determination}

According to the national standard $\mathrm{K}_{2} \mathrm{Cr}_{2} \mathrm{O}_{7}$ method, the CODcr of all adsorbed and desorbed solutions was determined ${ }^{[15]}$.

\subsection{Dynamic adsorption of resin}

At room temperature, benzoic acid and sorbic acid solutions were passed through the adsorption column containing $5.00 \mathrm{~mL} \mathrm{ZH}-01$ resin at a certain flow rate to measure the concentration of benzoic acid and sorbic acid of each grade in the effluent. The dynamic adsorption curves of benzoic acid and sorbic acid were made respectively, and the optimal adsorption process conditions were determined. 


\subsection{Resin desorption conditions}

The desorption conditions of adsorbed saturated resins were tested under the conditions of different proportion of ingredients, different temperature and different flow rate, so as to determine the best process conditions for desorption.

\section{Results and discussion}

\subsection{Maximum absorption wavelength}

The maximum ultraviolet absorption wavelength of benzoic acid and sorbic acid was determined by 1.2 method, as shown in Fig. 1-2.

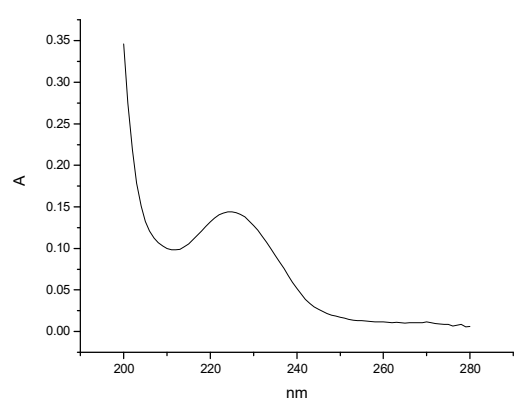

Fig.1 Ultraviolet absorption curve of benzoic acid

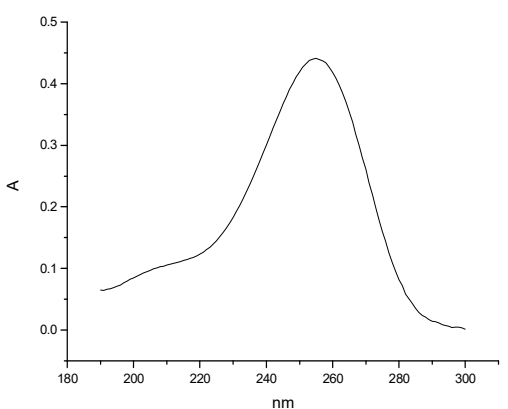

Fig. 2 Ultraviolet absorption curve of sorbic acid

\subsection{Standard curve drawing}

The standard curve of benzoic acid and sorbic acid solution was determined by 1.3 method, and the corresponding standard curve was drawn, as shown in Fig. 3-4. The curve was fitted by the least square method, and the standard curve equation was obtained. The standard curve equation of benzoic acid is $y=0.0671 \mathrm{x}+0.0087, \mathrm{R}^{2}=0.9998$. The standard curve equation of sorbic acid was $\mathrm{y}=0.2148 \mathrm{x}+0.0072, \mathrm{R}^{2}=1.0000$.

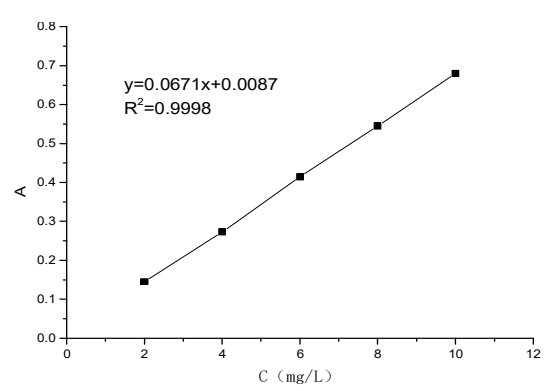

Fig.3 Linear relationship between benzoic acid concentration and absorbance

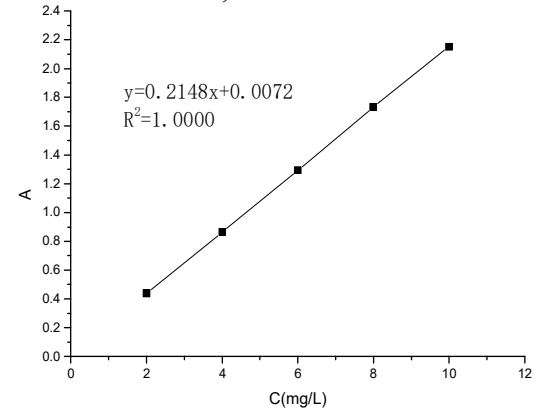

Fig.4 Linear relationship between sorbic acid concentration and absorbance

\subsection{Influence of flow rate on dynamic adsorption of resin}

The dynamic adsorption experiments were carried out at the flow rates of $2 \mathrm{BV} / \mathrm{h}, 4 \mathrm{BV} / \mathrm{h}$ and $6 \mathrm{BV} / \mathrm{h}$ according to the 1.5 method. The dynamic adsorption curves of benzoic acid and sorbic acid at different flow rates were determined by UV. See Fig.5-6 for their dynamic adsorption curves. It can be seen from Fig. 5-6 that the adsorption capacity of the resin will increase with the slow adsorption flow rate of waste water, and the treated waste water will also increase. This is because the benzoic acid and sorbic acid molecules can fully conduct membrane diffusion and particle diffusion with the resin bed as the flow rate of waste water slows down. In addition, the above dynamic adsorption data were also tested for CODcr by 1.4 method, and the dynamic adsorption curve was shown in Fig. 7. As can be seen from Fig. 7, with the difference of adsorption velocity, CODcr in wastewater also presents a certain degree of change. Therefore, a moderate adsorption flow rate of $4 \mathrm{BV} / \mathrm{h}$ was selected based on the above conditions. 


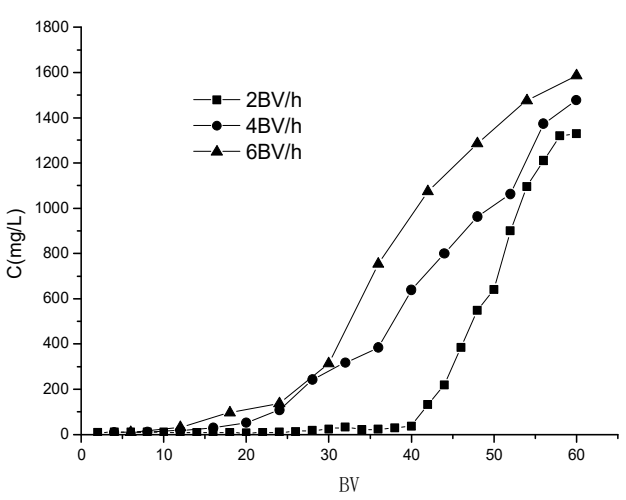

Fig. 5 Dynamic adsorption curve of benzoic acid at different flow rates

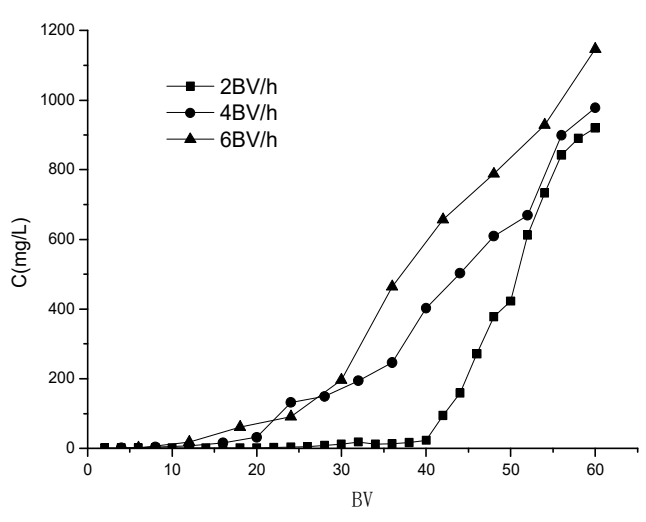

Fig. 6 Dynamic adsorption curve of sorbic acid at different flow rates

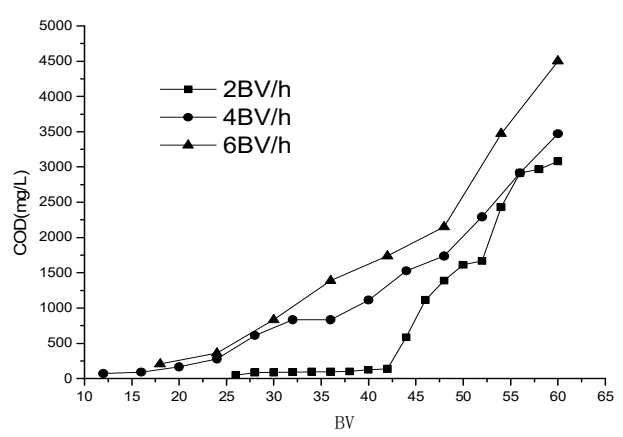

Fig.7 CODcr change curves at different flow rates

\subsection{Determine the wastewater treatment capacity}

The temperature is $25^{\circ} \mathrm{C}$, the velocity of $4 \mathrm{BV} / \mathrm{h}$ solution through attached under the condition of constant temperature circulating water jacketed adsorption column, from top to bottom to make it through the adsorption resin layer, to stop the adsorption initial concentration 5\%. The dynamic adsorption curve of the resin was shown in Fig. 8. According to figure 8, when the wastewater treatment capacity of resin reaches about $10 \mathrm{BV}$, the adsorption rate of benzoic acid and sorbic acid can reach $94.74 \%$ and $94.9 \%$ respectively. Meanwhile, the removal rate of CODcr reached $93.2 \%$. Therefore, we can conclude that the better treatment capacity of ZH-01 resin for wastewater containing benzoic acid and sorbic acid is $10 \mathrm{BV}$.

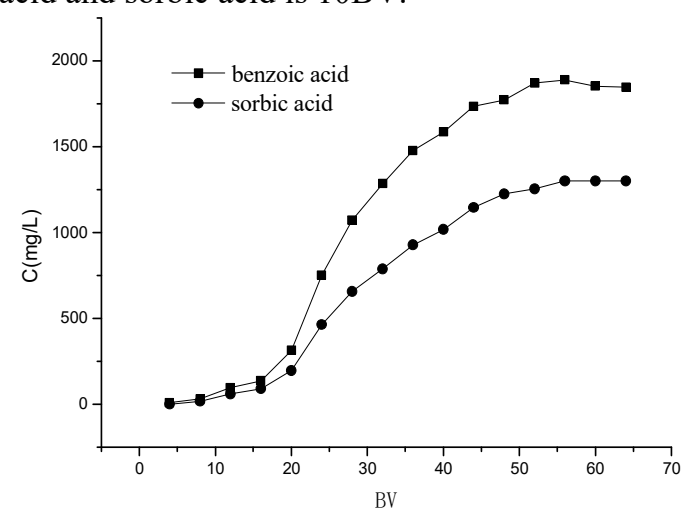

Fig. 8 Dynamic adsorption curves of ZH-01 resin for benzoic acid and sorbic acid

\subsection{Influence of desorption agent flow rate on resin desorption}

Under the condition of $45^{\circ} \mathrm{C}$, with $8 \% \mathrm{NaOH}$ as stripping agent, respectively by $\mathrm{BV} / \mathrm{h} 1,2, \mathrm{BV} / \mathrm{h}, 3 \mathrm{BV} / \mathrm{h}$ of stripping experiment was carried out on the resin flow velocity, and the stripping curve as shown in Fig. 9, as shown in figure 10 and figure 11. As can be seen from Fig. $9-10$, the desorption velocity is the best at 2BV/h, and as can be seen from fig. 11 , the removal rate of CODcr is also the highest at $2 \mathrm{BV} / \mathrm{h}$. Therefore, taking the above factors into consideration, the $2 \mathrm{BV} / \mathrm{h}$ velocity is adopted for desorption. 


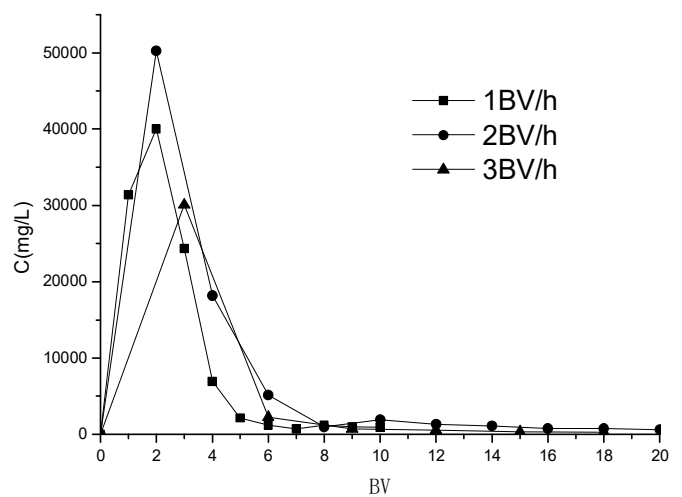

Fig.9 Desorption curve of benzoic acid at different flow rates

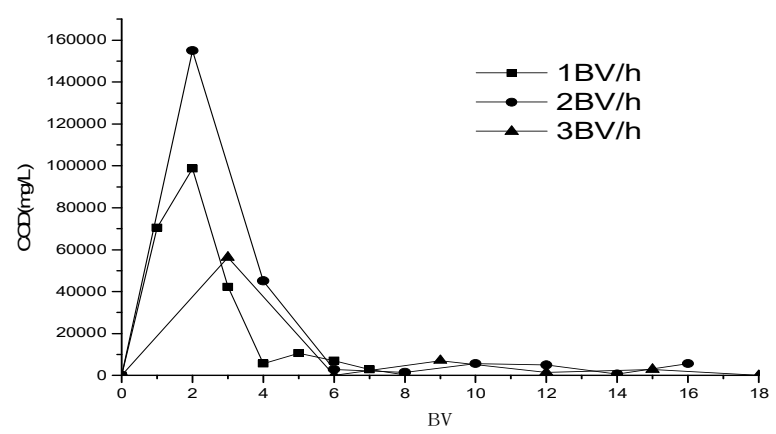

Fig. 11 Changes of CODcr at different flow rates

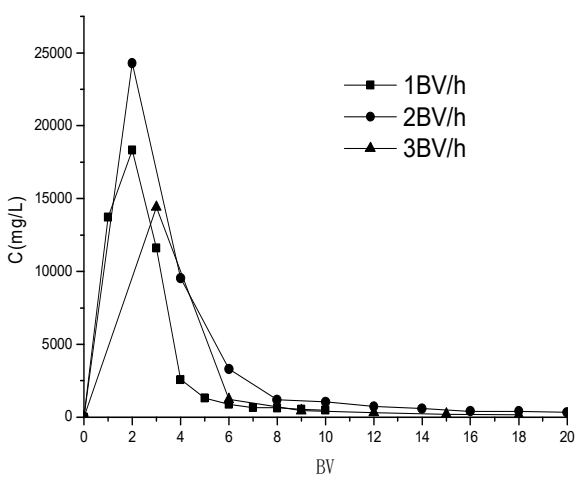

Fig. 10 Desorption curves of sorbic acid at different flow rates

\subsection{Influence of desorption temperature on dynamic desorption of resin}

With $1 \mathrm{BV} / \mathrm{h}$ stripping velocity, with $8 \% \mathrm{NaOH}$ solution as resin stripping agent, in $30^{\circ} \mathrm{C}, 45^{\circ} \mathrm{C}$ and $60^{\circ} \mathrm{C}$ under three different temperature dynamic stripping experiment, stripping curve as shown in Fig. 12-14. Fig. 12-14 showed that rise in the temperature of the stripping become more concentrated, and useful $\mathrm{ZH}-01$ resin regeneration performance is good. This is because the stripping process is an endothermic process, to improve the temperature of the stripping, can make stripping agent molecules of the resin micro channel, increased resin pore benzoic acid and sorbic acid molecules and stripping agent molecules collide, is advantageous to the reaction, so you can choose the best temperature of $60^{\circ} \mathrm{C}$ as stripping.

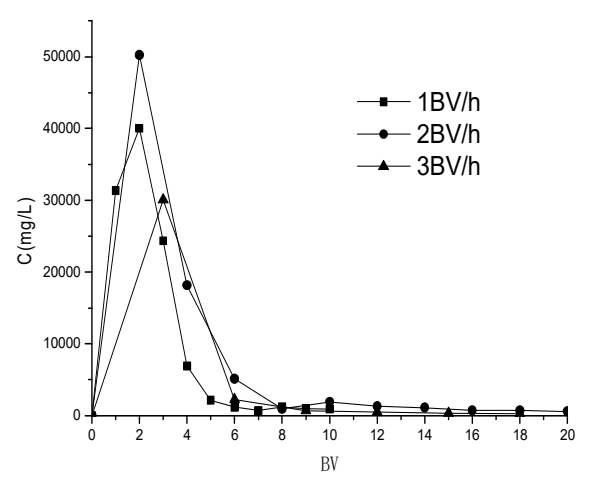

Fig. 12 Desorption curves of benzoic acid at different temperatures

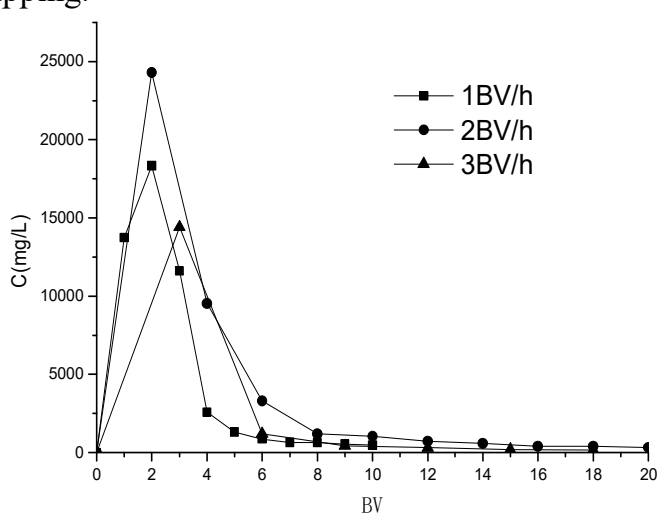

Fig. 13 Desorption curves of sorbic acid at different temperatures 


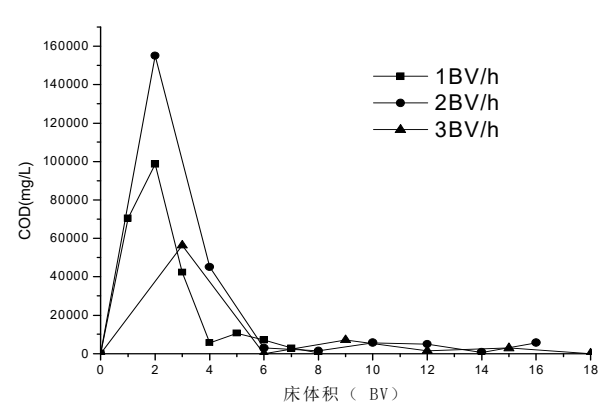

Fig. 14 Changes of COD at different temperatures

\subsection{Influence of the ratio of desorption agent on the resin desorption performance}

This experiment chooses dilute $\mathrm{NaOH}$ solution stripping was studied for the resin, the flow rate of $1 \mathrm{BV} / \mathrm{h}$, under the condition of temperature $45{ }^{\circ} \mathrm{C}$, respectively by $2 \% \mathrm{NaOH}$ solution and $4 \% \mathrm{NaOH}$ solution and $8 \% \mathrm{NaOH}$ solution as stripping agent, $\mathrm{ZH}-01$ resin for adsorption of benzoic acid and sorbic acid solution has been saturated with useful ZH- 01 resin stripping experiments, stripping curve as shown in Fig. 15 - 17. It can be seen from Fig. 15 and Fig. 17 that $8 \% \mathrm{NaOH}$ solution as a desorption agent has a good desorption effect on benzoic acid and sorbic acid.

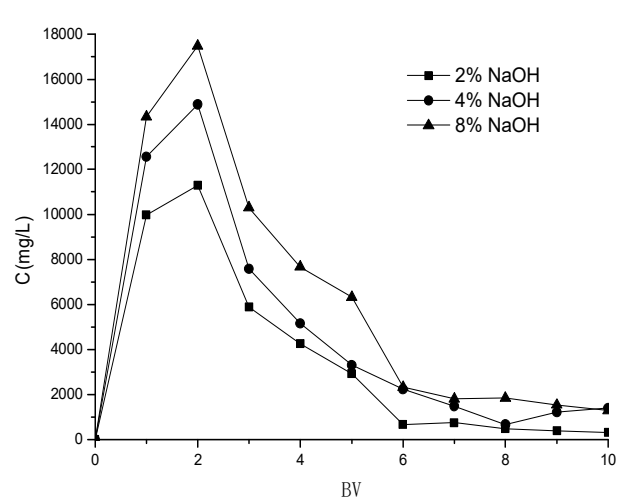

Fig.15 Desorption curve of p-benzoic acid with different desorption ratios

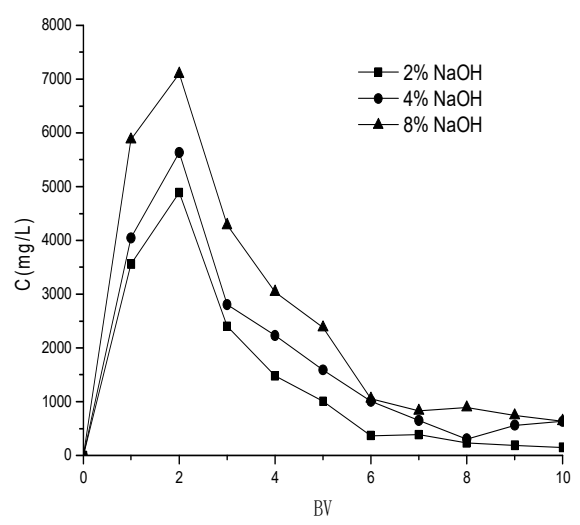

Fig. 16 Desorption curves of sorbic acid with different desorption ratios

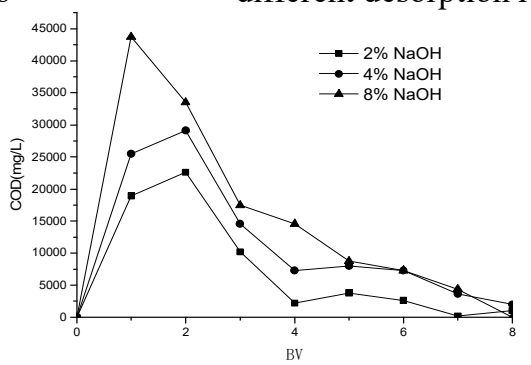

Fig..17 Change curve of CODcr with different proportion of desorption agent

\section{Conclusion}

The CODcr of the original water sample containing benzoic acid and sorbic acid was $19115 \mathrm{mg} / \mathrm{L}$, the average adsorption rate was $94.05 \%$ when the water sample was treated with $\mathrm{ZH}-01$ resin to reach $10 \mathrm{BV}$, and the CODcr of the outflow water sample was reduced to $1684 \mathrm{mg} / \mathrm{L}$, so the removal rate of CODcr reached $91.2 \%$. When $\mathrm{NaOH}$ is used as resin desorption agent, the final average desorption rate can reach $95.3 \%$. ZH-01 resin not only has good adsorption and desorption performance, but also can get high concentration of benzoic acid and sorbic acid, which has achieved the purpose of separation and recovery, and the recovery and utilization of resources. Processing conditions are as follows: Absorption: the amount of waste water processing $10 \mathrm{BV} / \mathrm{batch}$, and waste water flow rate is $4.0 \mathrm{BV} / \mathrm{h}$, the temperature is $25^{\circ} \mathrm{C}$. Stripping: stripping agent and the use of $8 \% \mathrm{NaOH}$ solution, velocity of flow control in $2.0 \mathrm{BV} / \mathrm{h}$, the temperature at $45^{\circ} \mathrm{C}$, optimization of adsorbent in about $10 \mathrm{ml}$ volume. Through the above simulation experiment, we can apply it to the treatment of benzoic acid and sorbic acid production waste water, and at the same time recover high concentration of benzoic acid and sorbic acid, so as to realize the recycling of resources, and provide sufficient theoretical basis for enterprises. 


\section{Acknowledgement}

The research is supported by a Project Funded by Innovation and entrepreneurship training program for college students in Jiangsu province (2019).

\section{References}

[1]Gui-qin Zhu, Na Zhao, Fen-fen Guo. Treatment of benzoic acid in wastewater with H-103 macroporous adsorbent resin [J]. Industrial water treatment,2010,30(4):63-65.

[2] Jing-ping Wang, Jian Chen, Zheng-hao Fei, Xing-long Wang, Yu-feng Sun, Wei-hua Tao, RONG Xur. Adsorption properties of ZH-01 resin for benzoic acid [J]. Chemical world, 2011,66(10):580-583,598.

[3] Kong W S, Ju T J, Park J H, et al. Short and characterization of hydrogenated sorbic acid grafted dicyclopentadiene tackifier. International Journal of Adhesion and Adhesives, 2012, 38 (10) : 38-44

[4] Xiao-qing Qian, Rong-ping Ji, Jun-jie Ji, Cheng-da He. High concentration methylbenzoic acid production wastewater treatment project example [J]. Industrial water treatment,2011,31(6):80-82.

[5] Xiao-yan Jiao, Gui-qin Zhu, Na Zhao, Chang-qing Wang. Study on adsorption of benzoic acid by NKAIImacroporous resin in water[J]. Journal of hebei north university (natural science edition), 2011, 28 (1) : 2125.

[6] Gui-qin Zhu, Xiao-yan Jiao. Study on treatment of simulated benzoic acid wastewater by macroporous adsorption resin [J]. Journal of hebei north university (natural science edition),2009,26(4):14-17.

[7] Shu-he Tang, Zheng-hao Fei, Jian Chen, Zhong-tang Liu. Thermodynamic and kinetic characteristics of static adsorption of 2-aminopyridine by o-aminophenol modified adsorption resin. Journal of ion exchange and adsorption, 2012,28(2):150-157.

[8] Yu-feng Sun, Zhong-tang Liu, Zheng-hao Fei,,Zheng-xing Li,Jing-ping Wang.Adsorption of p-phenol and pnitrophenol on adsorption resin modified by trimellitic anhydride [J]. Journal of computer \& applied chemistry,2013,30(12):1457-1460.

[9] Chai K G, Ji H B, Dual functional adsorption of benzoic acid from wastewater by in - -based chitosan grafted B - cyclodextrin. Journal of Chemical Engineering, 2012, 203 (9) : 309-318

[10]Pei Zhao, Yi Pan, San-san Zhao. Adsorption of o-nitroaniline wastewater by macroporous resin [J]. Journal of guangdong chemical industry,2012,40(4):131-132.

[11] Ma Y, Zhou Q, Li A M, et al. Preparation of A will be magnetic microporous adsorbent and its adsorption behaviors of $p$ - nitrophenol and hlorotetracycline. Journal of Hazardous Materials, 2014266 (2) : 84-93

[12] Guang-hui Qi, chun-yan Ding, Kun Zhou, Jing Li. Application of macroporous adsorption resin in organic wastewater treatment [J]. Journal of Hebei chemical industry, 2009,32 (4):69-71.

[13] Shu-peng Li, Hu Fang, Xue-song Li, Wei-chang Xu, Hai-sheng Yan. Experimental study on treatment of industrial wastewater containing nitrobenzene by macroporous resin adsorption [J]. Journal of Industrial water and wastewater,2011,42(5):32-37.

[14] Mi Wei, Chao Li, Zhong-dong Liu. The detection of benzoic acid as a preservative in condiments [J]. Journal of china food additives, 2012,5(2):199-204.

[15] Dan-li Xi, Yu-sheng Sun, Xiu-ying Liu. Environment and monitoring [M], Beijing: higher education press, 1998. 